\title{
LAS MARCAS Y EL PROCESO DE SOCIALIZACIÓN DEL CONSUMO EN NIÑOS Y ADOLESCENTES: EL CASO COLOMBIANO1
}

Oscar Javier Robayo Pinzón²

Politécnico Grancolombiano

osrobayo@poli.edu.co

Material original autorizado para su primera publicación en la revista académica REDMARKA. Revista Digital de Marketing Aplicado.

http://doi.org/10.17979/redma.2012.02.08.4760

Recibido: 20 Enero 2012

Aceptado 1 Junio 2012

\section{RESUMEN}

El estudio de los niños como consumidores ha sido de gran interés para los investigadores a lo largo de varias décadas. El presente artículo presenta en primer lugar una revisión de las principales tendencias y temas de interés en la investigación del público infantil y adolescente, al tiempo que propone una metodología alternativa que incluye el Análisis de Datos Textuales como una forma de aproximación a las tipologías lingüísticas que caracterizan diferentes grupos de edad. Se aplicó además una encuesta a una muestra de 1050 participantes. Los resultados indican que las marcas relacionadas con categorías

\footnotetext{
${ }^{1}$ El proyecto de investigación del cual es producto este artículo de investigación, ha sido financiado por la Fundación Politécnico Grancolombiano, mediante el contrato de investigación No: 2011FMCA-MI2-TC-31 del 7 de marzo de 2011.

2 Docente Investigador del Departamento Académico de Mercadeo y Publicidad. Facultad de Mercadeo, Comunicación y Artes. Institución Universitaria Politécnico Grancolombiano. Psicólogo, y Magíster en Psicología del Consumidor, de la Fundación Universitaria Konrad Lorenz. Catedrático e investigador con experiencia en investigación básica y de mercados, principalmente a nivel cualitativo. Actualmente es docente de las materias de Comportamiento del Consumidor, Investigación del Consumidor, Investigación de Mercados I, dentro del programa de Mercadeo y Publicidad del Politécnico Grancolombiano. Es docente de otras universidades (FUKL, Universidad de Bogotá Jorge Tadeo Lozano, Universidad EAN, Universidad Piloto de Colombia) a nivel de pregrado y posgrado, así como investigador free lance a nivel cualitativo y cuantitativo para diferentes empresas.
}

REDMARKA UIMA-Universidad de A Coruña - CIECID

Año V, Número 8, (2012), v2 pp. 21-51 http://www.redmarka.org/ 
de tecnología e internet tienen un alto nivel de conciencia y su presencia contribuye a mediar en los procesos de socialización particularmente con sus pares (amigos) en el caso de los adolescentes (edades entre 13 y 17 años). Para los niños de edades entre 9 y 12 años, la relación con sus padres continúa siendo fuerte y estrecha, en particular en actividades relacionadas con el consumo, tales como el realizar compras en supermercados y ver programas de televisión como novelas y noticias.

Palabras clave: niños, socialización del consumo, análisis de datos textuales.

\section{ABSTRACT}

TRADEMARKS AND THE CONSUMER SOCIALIZATION OF CHILDREN AND TEENAGERS: THE COLOMBIAN CASE

The study of children as consumers has been of great interest to researchers over several decades. This article first presents a review of major trends and issues in research on child and adolescent audiences while proposing an alternative methodology that includes textual data analysis as a way of approaching linguistic typologies that characterize different age groups. Was also carried out a survey of a sample of 1050 participants. The results indicate that brands related to categories of technology and the Internet have a high level of awareness and their presence helps to mediate the processes of socialization especially with their peers (friends) in the case of adolescents (ages 13 and 17 years ). For children aged 9 and 12 years, the relationship with their parents remains strong and narrow, particularly in consumer-related activities such as shopping in supermarkets and watch TV shows like soap operas and news.

Key Words: kids, consumer socialization, textual data analysis. 


\section{Introducción}

Dentro de la historia de la investigación del consumidor, los niños han sido estudiados y considerados como un segmento de interés desde mediados de la década de los 50, cuando aparecieron los primeros artículos sobre temas como el consumo conspicuo y la fidelidad a la marca (John, 1999). Sin embargo, no fue sino hasta mediados de la década de los 70 que la investigación sobre los niños como consumidores se consolidó como un área de estudio importante y tuvo una mayor relevancia dentro de la comunidad del mercadeo y la publicidad. En esa década los intereses de investigación estuvieron enfocados principalmente en la influencia que tiene la televisión en el comportamiento infantil (Chandler and Heinzerling, 1999). Durante las décadas de los 80 y 90, a medida que la capacidad de compra de los niños iba en aumento a nivel global, los practicantes del mercadeo fueron redefiniendo sus intereses de investigación para el segmento infantil (Solomon, 1991; Pecora, 1995). Durante este mismo período de tiempo, la investigación de los niños como consumidores se incrementó en gran medida tanto en cantidad como en volumen. El eje conceptual que ha guiado una parte importante del desarrollo de estas investigaciones es la consideración de la infancia y adolescencia como un conjunto de etapas sucesivas en las cuales se va dando una evolución progresiva en las capacidades cognitivas tanto analíticas como perceptuales, dando como resultado un consumidor que ya en su período de adultez debería poseer un proceso de decisión de compra más elaborado y racional (Robayo, 2011).

\section{Revisión de la Literatura}

Tal como lo menciona John (1999), el período comprendido entre el nacimiento y la adolescencia contiene una serie de cambios importantes en las funciones cognitivas y la maduración social. Los niños desarrollan destrezas que les permite ir más allá del nivel perceptual, generando un pensamiento más abstracto acerca 
de su entorno físico y social. Así mismo, adquieren nuevas herramientas para procesar información, lo cual les permite organizar y utilizar más rápidamente lo que aprenden a través de las experiencias que tienen en diferentes contextos de consumo. Este desarrollo también les permite tener un mejor entendimiento de las relaciones interpersonales, lo cual les facilita ver las situaciones a través de múltiples perspectivas.

A pesar del interés creciente en el estudio de los niños como consumidores que se describió con anterioridad y del crecimiento de la capacidad de compra de este segmento, algunos autores señalan que aspectos como la forma en la cual se relacionan los niños con los productos, el cómo llegan a establecer preferencias y cuál es el valor de marca percibido por ellos, resultan ser áreas relativamente inexploradas por parte de la comunidad científica del mercadeo (Chandler y Heinzerling, 1999). Resulta interesante por lo tanto, abordar el estudio de la relación que existe entre las marcas y el público infantil y adolescente. En este sentido, cabe mencionar que la marca ha servido tradicionalmente para añadir dimensiones a los productos logrando diferenciarlos de otros productos diseñados para satisfacer las mismas necesidades (Keller, 1998). Una de las aproximaciones típicas para establecer el valor de la marca se centra en el diferencial de precio que los consumidores están dispuestos a pagar por una marca específica por encima de otras opciones que se encuentren disponibles en una categoría de producto (Grassl, 1999). A partir de lo anterior, se abre la discusión acerca de cuál puede llegar a ser una alternativa más cercana para la interpretación de la relación entre las marcas y el público infantil, ya que como lo señalan varios estudios, este vínculo parece que empieza a establecerse desde muy temprana edad. Los resultados indican que incluso desde los seis meses de edad, los bebes son capaces de formar imágenes mentales de logos corporativos y personajes característicos de algunas marcas (McNeal y Yeh, 1993). A los tres años de edad, uno de cada cinco niños ya realiza peticiones directas a sus padres de productos con nombres de marca específicos (Center for a New American Dream, 2002). A donde quiera que vayan los niños, ya sea supermercados, restaurantes de

REDMARKA UIMA-Universidad de A Coruña - CIECID 
comidas rápidas, tiendas de juguetes o tiendas de ropa, se van a encontrar con personajes y productos licenciados de una gran cantidad de programas y páginas web con los cuales habrán interactuado durante muchas horas a través de la televisión y más recientemente, el internet (Weehsler, 1997). Esto ha llevado a que se desarrollen tácticas como el mercadeo "entre pares" (peer to peer marketing), en el cual algunas compañías de vestuario juvenil contactan adolescentes que tengan el perfil de agentes influenciadores para que promuevan entre sus amigos y compañeros el uso de ciertas marcas, creando de esta manera comunidades artificiales en torno a ellas (Wolff, 2003).

Esta nueva realidad del consumidor infantil hace necesario explorar las condiciones que sustentan la formación de sus preferencias, actitudes y conductas de compra. En este sentido, es importante mencionar que los patrones de compra característicos de los niños se forman y son afectados por un conjunto de agentes socializadores que incluyen a sus familias, amigos y compañeros de escuela, así como los medios de comunicación que consumen (Ward, 1974; Moschis and Moore, 1979; Moschis, 1981; Ozgen, 2003). Estos agentes tienen una importancia crítica en el proceso de aprendizaje de los niños y adolescentes ya que con mucha frecuencia determinan el hecho de que un niño adquiera o no una determinada marca que se encuentre presente en el mercado. Al respecto cabe mencionar que existe un conjunto de factores que se interrelacionan entre sí para influir en el proceso de toma de decisiones de los niños, entre los cuales la familia se constituye como uno de los más importantes, dada la preponderancia que tienen en este ámbito los mecanismos de modelamiento por parte de los padres, así como la interacción directa entre padres e hijos (Sidin et al, 2008). Otros estudios refuerzan la noción de que los padres juegan un papel principal en los procesos de socialización hasta el comienzo de la adolescencia, momento en el cual los pares (amigos, compañeros) se convierten en la principal fuente de información en cuanto a comportamientos y preferencias de compra (Moschis and Moore, 1980). En estos estudios se identifica además a los padres como la fuente de información racional para la toma de decisiones de los niños, en tanto que los pares y medios

REDMARKA UIMA-Universidad de A Coruña - CIECID 
de comunicación se establecen como las fuentes de información irracional (Moschis and Churchill, 1978). La influencia de la familia en las decisiones de los niños es más fuerte para las categorías relacionadas con alimentos, productos de cuidado personal y artículos para el hogar, tales como muebles y juguetes, en tanto que los amigos y los medios de comunicación tienen una influencia marcada para los productos en que los niños invierten su ingreso discrecional, tales como dulces, vestuario y accesorios (Wimalasiri, 2004).

Al estudiar este tipo de temas, se ha logrado un avance importante en la conceptualización y la comprensión de los factores que afectan el desarrollo del conocimiento en los consumidores. Basados en los hallazgos provenientes de la investigación en los procesos de socialización, los investigadores han desarrollado marcos conceptuales que han permitido establecer los factores clave que influyen en la socialización del consumo (Churchill and Moschis 1979; Moschis and Moore 1979). Dentro de este marco conceptual se ha establecido la presencia de dos clases principales de factores: los ambientales y los cognitivos. Los factores ambientales tales como la familia, los pares (amigos y compañeros) y los medios masivos de comunicación afectan la adquisición de conocimiento sobre el consumo en los niños, modificando el tipo, cantidad y calidad de la información y experiencias de compra a los que están expuestos. Los factores cognitivos, compuestos por las habilidades cognitivas propias de cada etapa del desarrollo afectan la adquisición de conocimiento en los niños a través de la interpretación y la organización de las experiencias de consumo que se van acumulando a través del tiempo. En conjunto, estos factores se han establecido como los responsables, dentro del enfoque cognitivo de la investigación del consumidor, de moldear la forma en que los niños adquieren el conocimiento y las habilidades involucradas en los procesos de socialización del consumo (John, 2008).

Sin embargo, algunos autores sugieren más recientemente que este modelo tradicional debe empezar a ser cuestionado a la luz de los cambios sociales, económicos, culturales y tecnológicos que se han dado en las últimas dos 
décadas (Dotson and Hyatt, 1994; 2000). El cambio más notable se refiere al protagonismo que han empezado a tener los preadolescentes en las decisiones familiares al tiempo que están efectuando más compras de productos de forma independiente a edades cada vez más tempranas (McNeal and Yeh, 1993). Estos cambios recientes sugieren que existe una necesidad cada vez mayor de realizar estudios más amplios en este segmento poblacional en países en vías de desarrollo. Esto es particularmente cierto si se tiene en cuenta que los niños y adolescentes de hoy pasan más tiempo en entornos comerciales que las generaciones anteriores, al tiempo que son impactados perceptualmente a través de muchos más medios de comunicación que los que existían hace apenas un par de décadas (Weehsler, 1997). El ir de compras, como un factor que puede influir en los jóvenes consumidores, no ha sido estudiado adecuadamente desde un enfoque de corte más empírico, a pesar de que ahora parece ocupar mucho más tiempo en la agenda de actividades de este público juvenil. Los niños de 10 años van en planes de compras (en muchas ocasiones acompañando a sus padres) alrededor de 250 veces al año (Dotson and Hyatt, 1994). Los niños colocan esta actividad como se segunda favorita, después de ver televisión (Schulman and Clancy, 1992). Estos resultados deben ser revisados a su vez a la luz de la entrada y la creciente penetración que han tenido en la población infantil y adolescente los nuevos medios digitales como el internet y la comunicación móvil. Además de estos elementos se debe tener en cuenta que para el mercado infantil también parece estar presentándose el fenómeno conocido como fragmentación, el cual consiste en una división natural de los mercados homogéneos de hace 20 años o más, en mercados mucho más diferenciados, con segmentos más especializados que han generado en las empresas una serie de innovaciones, entre las cuales se cuentan productos y campañas de comunicación más enfocadas y "personalizadas" (Shimp, 2000). Sin embargo, es posible que el factor más relevante en la conformación de los patrones de consumo y de preferencias en los jóvenes sea precisamente la marca. El incremento en las comunicaciones y la presencia de las marcas en la vida cotidiana de los niños ha hecho que no solo 
exista un mayor conocimiento sino cada vez más preferencias específicas por ciertas marcas a edades cada vez más tempranas (Dotson and Hyatt, 2005). En este nuevo contexto, los niños y adolescentes están recibiendo una influencia que proviene no sólo de los esfuerzos de comunicación de las marcas sino también de otros agentes que han sido catalogados igualmente por algunos autores como de carácter "irracional", en particular, los modelos aspiracionales para el consumo de ciertas marcas que los niños observan en sus pares (peers) a través de mecanismos de presión social (Dotson and Hyatt, 1994). A este fenómeno se le debe añadir el hecho de que los niños cada vez comparten menos tiempo con sus padres y reciben simultáneamente más comunicación alternativa por parte de las marcas, tales como el apoyo (endorsement) que logran establecer con estrellas deportivas, musicales y de otras industrias del entretenimiento. Esta nueva realidad a la que están expuestos los niños y adolescentes ha hecho que se desarrollen en ellos patrones de comportamiento con respecto a las marcas en los que prima la preferencia por aquellas que les permita reafirmar su propia individualidad así como el poder expresar un mensaje a la sociedad (Public Broadcasting Service, 1999). Es a partir de estas tendencias recientes que dentro de este artículo se plantea una problemática relacionada con el nivel de conciencia e imagen de las marcas y los factores psicosociales y comunicacionales que están asociados al aprendizaje de las mismas en los niños y adolescentes de 9 a 17 años de edad en el contexto de un país en vías de desarrollo como es la ciudad de Bogotá, Colombia.

Estos hallazgos aportarán evidencia adicional con respecto al rol que cada uno de estos factores puede estar teniendo en el proceso de socialización del consumo en este segmento poblacional.

\section{Hipótesis}

Teniendo en cuenta las condiciones rápidamente cambiantes de la sociedad en la que están desarrollándose los niños y adolescentes en la actualidad, resulta adecuado explorar las posibles relaciones que puedan existir entre algunas

REDMARKA UIMA-Universidad de A Coruña - CIECID 
variables clave que median la forma en la que este grupo poblacional está generando sus procesos de socialización del consumo. Específicamente, se plantean las siguientes hipótesis de trabajo:

$\mathrm{H} 1$ : Existe una asociación significativa entre el rango de edad y las actitudes hacia los padres y las marcas en diferentes categorías de producto.

H2: Existe una asociación significativa entre el nivel socioeconómico y las actitudes hacia las marcas en diferentes categorías de producto

H3: Existe una diferencia significativa en la percepción que tienen los niños y adolescentes con respecto a la influencia que tienen sus amigos frente a sus padres en su comportamiento global.

H4: Existe una asociación significativa entre el rango de edad y la percepción que tienen los niños y adolescentes con respecto a la influencia que tienen sus amigos frente a sus padres en su comportamiento global.

\section{Metodología}

\subsection{Instrumentos}

\subsubsection{Fase cualitativa}

Para llevar a cabo la fase cualitativa, se realizó un conjunto de sesiones de grupo a las que se denominó "Brands Insights", cuyo objetivo consistió en establecer en la primera parte de cada sesión un conjunto de categorías de producto relevantes para los participantes de acuerdo a la importancia que tienen ciertos productos en su estilo de vida, así como identificar el conjunto de marcas dentro de estas categorías que generaran el mayor interés, debate y respuesta por parte de los participantes. Una vez se establecieron las categorías de producto relevantes para los participantes, se aplicó una segunda parte dentro de cada sesión, cuyo objetivo consistió en determinar las actitudes, creencias y percepciones que tienen los participantes sobre dichas marcas. Las sesiones de grupo se aplicaron a los 
dos grupos de edad y a los tres niveles socioeconómicos establecidos para el estudio. Un primer grupo abarcó los participantes de 9 a 12 años de edad, y el segundo grupo a aquellos de entre 13 y 17 años. Así mismo, se tuvieron en cuenta tres niveles socioeconómicos: medio bajo, medio y medio alto. En cada sesión se trató de mantener una presencia equitativa entre los dos géneros, masculino y femenino. Se realizaron 20 sesiones de grupo en total, de acuerdo a la distribución y el número de participantes que se observa en la Tabla 1. A partir de los datos textuales hallados en cada una de las sesiones, se procedió a realizar la respectiva tabulación de los mismos en el software (SPAD: Système Portable pour l'Analyse de Données).

\subsubsection{Fase cuantitativa}

Para la recolección de datos en la fase cuantitativa se diseñó un instrumento de carácter estructurado, que tuvo como objetivo el obtener datos relacionados con la percepción y actitudes que tienen los participantes con respecto a la forma en la que realizan sus compras, cómo se conforma sus ingresos y las fuentes de las cuales los obtienen, cómo distribuyen su ingreso en aquellas categorías que resultaron relevantes a partir de los hallazgos obtenidos en la etapa cualitativaexploratoria mencionada en el apartado anterior, con quienes les gustaría ir a comprar ciertos productos (padres, amigos, o ellos solos), así como sus actitudes frente a los diferentes factores influenciadores hallados en la revisión de la literatura en el área: padres, amigos (pares) y medios de comunicación. En esta última sección se realizó una adaptación de la escala de medición de actitudes elaborada y validada por Dotson y Hyatt (2005) con una opción de respuesta dicotómica: SI o NO. Los 13 ítems aplicados aparecen a continuación de acuerdo a la categorización de factores diseñada originalmente por los autores mencionados:

Amigos y compañeros (peers) 
- Me siento preocupado cuando mis amigos tienen un producto que yo no puedo tener.

- Lo que piensan mis amigos es más importante que lo que piensan mis papas.

- Prefiero compartir tiempo con mis amigos en lugar de hacerlo con mis papas.

Padres:

- Mis padres y yo estamos de acuerdo en la ropa que debo usar

- Lo que piensan mis papas es más importante que lo que piensan mis amigos.

Compras:

- Cuando se trata de ropa, yo casi siempre se lo que voy a comprar antes de salir de casa.

- Para mí es muy importante tener ropa de marca.

Medios de Comunicación

- Ver TV es la actividad más divertida que hago en casa.

- Siempre que puedo, prefiero ver TV que navegar en Internet.

- Siempre que puedo, prefiero navegar en internet en lugar de ver TV.

- Me contacto más con mis amigos a través de Internet que de manera personal.

- Cuando entro a internet tengo claro por cuanto tiempo voy a navegar.

- Tengo acceso a Internet cada vez que quiero usarlo. 


\subsubsection{Muestra}

La población objetivo abarca los niños y adolescentes de 9 a 17 años de edad, de ambos géneros, de nivel socioeconómico medio bajo, medio y medio alto de la ciudad de Bogotá D.C. (Colombia). En cuanto al tamaño de la muestra, para la fase cualitativa, el tamaño total fue de 160 niños y adolescentes, de los cuales el $50 \%$ fueron de género masculino y $50 \%$, femenino (ver Tabla 1).

\section{Tabla 1: Tamaño de la muestra fase cualitativa}

\begin{tabular}{|c|c|c|c|c|}
\hline & \multicolumn{3}{|c|}{ Nivel Socioeconómico } \\
\hline & & Medio Bajo & Medio & Medio Alto \\
\hline \multirow{2}{*}{ Edad } & De 9 a 12 & $\begin{array}{l}3 \text { sesiones (24 } \\
\text { participantes) }\end{array}$ & $\begin{array}{l}4 \text { sesiones (32 } \\
\text { participantes) }\end{array}$ & $\begin{array}{l}3 \text { sesiones ( } 24 \\
\text { participantes) }\end{array}$ \\
\hline & De 13 a 17 & $\begin{array}{l}3 \text { sesiones ( } 24 \\
\text { participantes) }\end{array}$ & $\begin{array}{l}4 \text { sesiones ( } 32 \\
\text { participantes) }\end{array}$ & $\begin{array}{l}3 \text { sesiones ( } 24 \\
\text { participantes) }\end{array}$ \\
\hline
\end{tabular}

Para la fase cuantitativa, la muestra tuvo un tamaño de 1050 niños y adolescentes. La muestra fue obtenida de forma no probabilística, por conveniencia a través de sujetos voluntarios. Los cuestionarios fueron aplicados de manera asistida en diferentes colegios (centros de enseñanza de nivel medio) de la ciudad de Bogotá. Antes de la aplicación de los cuestionarios se contó con la aprobación expresa por parte de al menos uno de los padres de cada participante. De la muestra de 1050 niños y adolescentes de la ciudad de Bogotá con los que se realizó la encuesta, el $47,2 \%$ son hombres y el $52,8 \%$ son mujeres, los cuales están actualmente en el colegio cursando desde $3^{\circ}$ a $11^{\circ} \mathrm{y}$ grado $12^{\circ}$ distribuidos de la siguiente manera, en tercer grado el $0.39 \%$, en cuarto grado el $3.96 \%$, en quinto el $4.93 \%$, en sexto el $12.85 \%$, en séptimo el $7.34 \%$, en octavo el $6.28 \%$, en noveno el $24.54 \%$, en decimo el $25.22 \%$, en once el $13.91 \%$ y grado 12 el $0.58 \%$, con lo que podemos concluir la mayoría de los encuestados eran de los grados superiores los cuales son $9^{\circ}, 10^{\circ}$ y $11^{\circ}$ con un $63.67 \%$ y el restante $36.33 \%$

REDMARKA UIMA-Universidad de A Coruña - CIECID 
estaban distribuidos en los grados de $3^{\circ}$ de primaria a $8^{\circ}$ de bachillerato, con la excepción del grado $12^{\circ}$ que tuvo solamente un $0.58 \%$ del total de la muestra.

\section{Resultados}

\subsection{Fase Cualitativa}

Con respecto a la primera y segunda hipótesis planteadas en relación con una posible asociación significativa entre el rango de edad y las actitudes hacia los padres y las marcas en diferentes categorías de producto, así como una posible asociación significativa entre el nivel socioeconómico y las actitudes hacia las marcas en diferentes categorías de producto, se llevó a cabo un Análisis de Correspondencias Simples dentro del método denominado Análisis de Tipologías Lingüísticas usando el software SPAD (Système Portable pour l'Analyse de Données).

Para ello, se comenzó por tabular la totalidad de los datos textuales provenientes de los verbatims (transcripciones textuales) de cada una de las sesiones de grupo en relación con diferentes categorías lingüísticas de interés. Estas categorías fueron seleccionadas a partir de las respuestas dadas por los participantes durante la primera parte de cada sesión. Las categorías que generaron mayor debate e interés en los niños y adolescentes y que por lo tanto fueron escogidas corresponden a:

1. Asociaciones y creencias con respecto a sus amigos.

2. Asociaciones y creencias con respecto al uso de internet.

3. Asociaciones y creencias con respecto a productos y servicios relacionados con entretenimiento.

4. Asociaciones y creencias con respecto a la tecnología.

5. Asociaciones y creencias con respecto a sus padres. 
Cada una de las sesiones dentro de la matriz de tabulación fue identificada con el rango de edad y el nivel socioeconómico respectivo de los participantes que hicieron parte de cada sesión.

El Análisis de Tipologías Lingüísticas permite a través del Análisis de Correspondencias Simples, establecer el perfil léxico que caracteriza a cada una de las categorías establecidas para las dos variables categóricas (nominales) que sean de interés para aportar evidencia en relación con la(s) hipótesis panteada(s). En este caso las variables categóricas corresponden a la edad (medida por rangos) y el nivel socioeconómico (3 niveles o estratos) de los participantes.

Al aplicar el Análisis de Correspondencias Simples, el software establece aquellas formas léxicas (palabras o frases -denominadas técnicamente "segmentos"-) que con mayor probabilidad caracterizan a cada categoría de cada variable que hace parte del análisis. Para el presente caso las variables analizadas con sus respectivas categorías corresponden a:

- Edad:

- De 9 a 12 años

- De 13 a 17 años

- Nivel Socioeconómico:

- Medio bajo

○ Medio

- Medio Alto

La primera parte del análisis implica la creación del diccionario de palabras, el cual se extrae de los verbatims de todas las sesiones de grupo aplicadas (Ver Tabla 2). El total de palabras obtenidas conforma el "corpus textual" sobre el cual se realiza el análisis de tipologías lingüísticas. 
Tabla 2 Diccionario de Palabras en SPAD

\begin{tabular}{|lr|}
\hline \multicolumn{2}{|c|}{ Creación del Diccionario de Palabras en SPAD } \\
\hline Número total de sesiones (verbatims) & 20 \\
\hline Número total de palabras & 2815 \\
\hline Número de palabras diferentes & 857 \\
\hline Porcentaje de palabras diferentes & $30 \%$ \\
\hline
\end{tabular}

Como se puede observar, el total de palabras obtenidas de los verbatims corresponde a 2815, de las cuales existen 857 palabras diferentes (aquellas que tienen una frecuencia de aparición de 2 o más veces dentro del corpus textual).

A continuación se realizó una depuración del diccionario de palabras, suprimiendo aquellas que tuvieran una frecuencia igual a 1. Esto con el fin de mantener solo aquellas que tuvieran dos o más menciones y que con mayor probabilidad fueran palabras "llenas", es decir, palabras que tienen verdadero significado dentro del contexto del estudio (ej: padres, amigos, páginas, Facebook, juegos, colegio, etc).

Después de realizar este procedimiento se obtuvo la Tabla de Contingencia Léxica, para lo cual se estableció la edad como variable de agrupación principal para los resultados (Ver Tabla 3).

Tabla 3. Conformación de la Tabla de Contingencia Léxica

\begin{tabular}{|c|c|c|c|c|c|}
\hline \multicolumn{6}{|c|}{ Conformación de la Tabla de Contingencia Léxica } \\
\hline Grupo & $\begin{array}{c}\text { Etiqueta de la } \\
\text { categoría }\end{array}$ & $\begin{array}{c}\text { Número de } \\
\text { sesiones }\end{array}$ & $\begin{array}{c}\text { Número de } \\
\text { formas léxicas }\end{array}$ & $\begin{array}{c}\text { Porcentaje } \\
\text { frente al total }\end{array}$ & $\begin{array}{c}\text { Número de formas } \\
\text { léxicas diferentes }\end{array}$ \\
\hline 1 & De 9a 12 & 10 & 1207 & 50,82 & 287 \\
\hline 2 & De 13 a 17 & 10 & 1168 & 49,18 & 255 \\
\hline \multicolumn{2}{|c|}{ TOTAL } & 20 & 2375 & 100 & 542 \\
\hline
\end{tabular}

Como se puede observar, cada rango de edad tuvo una participación muy cercana al $50 \%$ del total de formas léxicas dentro del corpus. 
Una vez se estableció el corpus textual depurado, se procedió a realizar el Análisis de Correspondencias Simples, el cual arrojó los resultados diagnósticos del modelo que se observan en la Tabla 4.

\section{Tabla 4. Análisis de Correspondencias Simple}

\begin{tabular}{|c|c|c|c|}
\hline \multicolumn{4}{|c|}{$\Lambda$ nálisis de Correspondencias Simples } \\
\hline Valores Propios \\
\hline \multicolumn{3}{|c|}{ Surna de los Valores Propios: 0,4050 } \\
\hline \multicolumn{4}{|c|}{ Histograma de los primeros 4 valores propios: } \\
Número & Valor Propio & Porcentaje & Porcentaje Acumulado \\
\hline 1 & 0,2009 & 49,60 & 49,60 \\
\hline 2 & 0,1053 & 26,01 & 75,61 \\
\hline 3 & 0,0988 & 24,39 & 100 \\
\hline 4 & 0 & 0,00 & 100 \\
\hline
\end{tabular}

Como resultado del procedimiento se obtuvo en primer lugar la tabla de valores propios (Ver tabla 4), en donde se encuentra que los factores 1 y 2 explican un $75,61 \%$ de la información hallada. Este porcentaje de información se considera adecuado para establecer conclusiones con respecto a las hipótesis planteadas.

En segundo lugar se obtuvo el plano de tipologías lingüísticas que aparece a continuación.

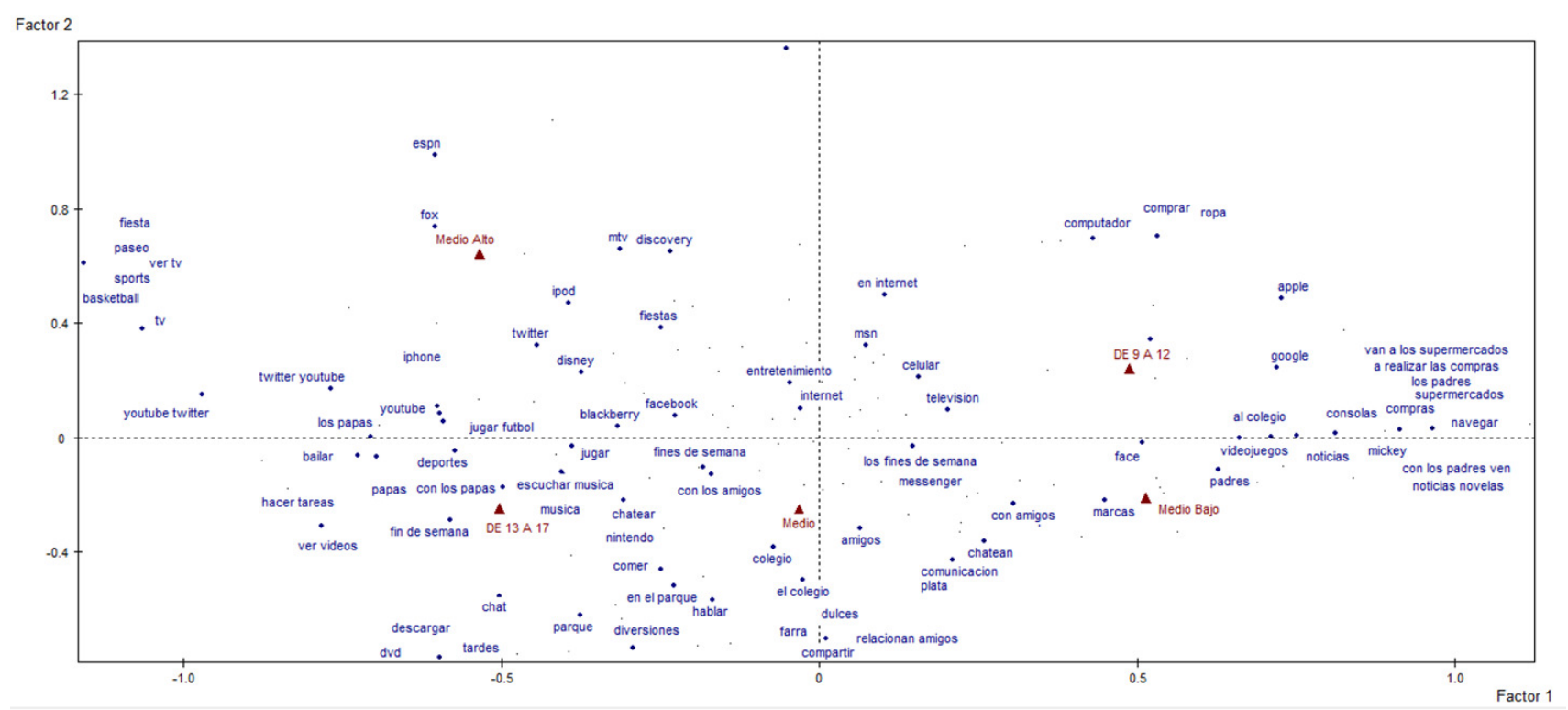

REDMARKA UIMA-Universidad de A Coruña - CIECID

Año V, Número 8, (2012), v2 pp. 21-51

http://www.redmarka.org/

ISSN 1852-2300 


\section{Figura 1. Mapa de Correspondencias: Análisis de Tipologías Lingüísticas para las variables Edad y Nivel Socioeconómico}

En el Mapa de Correspondencias se pueden observar en color azul, aquellas formas léxicas (palabras y frases -segmentos-) que resultaron tener una mayor contribución en los dos factores seleccionados. En color rojo aparecen las categorías correspondientes a las variables nominales: edad y nivel socioeconómico. La interpretación del mapa indica que aquellas formas léxicas que se encuentren más cercanas a una categoría, con mayor probabilidad representan las expresiones manifestadas por los participantes que pertenecen a dicha categoría. Aquellas formas léxicas que se encuentran en los extremos correspondientes a las zonas en donde se encuentran las categorías también hacen parte de la tipología lingüística de la(s) categoría(s) más cercanas. Las formas léxicas que se encuentran en el área cercana al origen del gráfico son comunes a todas las categorías de las variables analizadas.

De esta forma se puede observar que para la variable edad, existen tipologías lingüísticas bien establecidas para cada rango. En primer lugar, para el rango de 9 a 12 años, se observa que las actividades que más los caracterizan tienen que ver con las tareas relacionadas con la escuela ("al colegio"), el uso de videojuegos "consolas" y del computador. Una de las páginas que más se menciona en este grupo de edad es "Google", tal vez precisamente porque es su principal herramienta en la Web para desarrollar sus tareas escolares. Lo siguiente más característico en su perfil léxico es la importancia que tiene todavía en sus vidas la presencia de sus padres, la cual se ve reflejada principalmente en compartir dos tipos de actividades básicas en relación con la compra y consumo de productos: el ir a realizar compras con los padres a los supermercados ("van a los supermercados", "a realizar las compras", "compras", "supermercados"), y por otra parte, el compartir tiempo junto a sus padres para ver programas de televisión ("televisión", "noticias", "novelas"). Por otra parte se empieza a vislumbrar en este grupo el impacto de los nuevos medios de comunicación e interacción social 
basados en las nuevas tecnologías. Específicamente aquellas que les permiten tener contacto virtual en tiempo real con sus amigos a través de internet y de la mensajería "chat" de los teléfonos móviles ("con amigos", "chatean", "messenger", "face"). Con respecto al rango de edad de 13 a 17 años, se destaca en primer lugar el uso del internet, pero a diferencia del rango de edad de 9 a 12 años, este uso se amplía para "escuchar música", realizar descargas de videos, canciones y software de diferentes clases ("descargar"), hablar con los amigos ("chat", "chatear", "con los amigos", "Blackberry"), así como visitar páginas con contenidos más sociales y de entretenimiento ("Youtube", "Twitter", "Facebook", "ver videos"). También se destaca el hecho de que empiezan a mostrar actividades sociales fuera de casa, pero de manera casi que exclusiva con sus amigos. El compartir tiempo con los padres parece disminuir de manera drástica en este rango de edad. Se destacan el reunirse en los parques para hablar con sus amigos ("parque", "en el parque", "tardes", "hablar", "con los amigos"), y el practicar deportes en los parques ("deportes", "jugar futbol"). También se reconoce el mayor grado de independencia que tienen frente a sus padres, ya que parecen programar ellos mismos salidas dentro y fuera de la ciudad, acompañados de sus amigos, no de sus padres ("fiesta", "bailar", "paseo", "fines de semana"). Con respecto a las formas léxicas que comparten los dos grupos de edad, se destaca el "internet" muy cercano además a la palabra "entretenimiento", como las dos palabras que más caracterizan por igual a los participantes de todas las edades. Además, comparten el uso y la apropiación de los medios de comunicación con algunas marcas preferidas ("msn", "celular", "televisión"), y el compartir los fines de semana en actividades con sus amigos ("amigos", "compartir", "con los amigos").

Sobre la variable nivel socioeconómico, se destaca el hecho de que los participantes de clase media alta parecen tener una preferencia más clara por algunas marcas en diferentes categorías tales como canales de televisión ("Fox Sports", "ESPN", "MTV", "Discovery") y productos tecnológicos ("ipod", "iphone"). Los participantes de clase media muestran un perfil léxico muy cercano al origen del gráfico, por lo cual se puede decir que sus actividades e intereses giran en 
torno a la vida escolar y el compartir con sus amigos ("colegio", "amigos", "con los amigos", "el colegio"). Los participantes de nivel socioeconómico medio bajo están mucho más cerca del perfil léxico que indica una mayor influencia de los padres en sus actividades cotidianas ("padres", "van a los supermercados”, "con los padres ven").

De esta forma se puede observar que dependiendo del rango de edad y del nivel socioeconómico los factores externos tales como los padres, los amigos y los medios de comunicación tienen un mayor o menor nivel de presencia en el perfil léxico de cada subgrupo (categoría). Estas tipologías lingüísticas pueden ser de gran interés tanto para los profesionales de las ciencias sociales así como para los practicantes del mercadeo y la publicidad que estén buscando desarrollar una mejor comprensión de este segmento poblacional.

\subsection{Fase cuantitativa}

A partir de los resultados descriptivos hallados para las diferentes categorías de preguntas contenidas en la encuesta aplicada a la muestra de 1050 niños y adolescentes, se encontró lo siguiente:

\section{- Compras}

Se les pregunto a los participantes sobre las compras para identificar qué tipo de productos compran solos, cuales en compañía de sus padres y cuales productos compran sus padres, y se encontraron los siguientes resultados:

Los productos relacionados con comida (bebidas y papas fritas) en su mayoría lo compran solos o en compañía de sus padres, productos como el cine y el calzado deportivo lo compran/adquieren en su mayoría en compañía de sus padres, y productos de tecnología como el teléfono celular lo compran los padres solos en su mayoría.

\section{- Ingresos y Fuentes}


A los encuestados se les pregunto sobre si tenían fuentes de ingresos para realizar sus compras y sobre los tipos de fuentes que tenían pudiendo ser los padres, familiares, el trabajo propio (informal) u otra fuente de ingresos.

El $28.50 \%$ de los encuestados afirmó que no tenía una fuente de ingresos y la distribución de este porcentaje por género fue del $27.22 \%$ de los niños y el $26.66 \%$ de las niñas.

El $71.50 \%$ de los encuestados contestó que si tenían una fuente de ingresos y de las opciones antes mencionadas los encuestados respondieron que las fuentes de las que mas obtienen dinero son los padres (82.74\%) y familiares (30,94\%).

Las fuentes de las que menos obtienen dinero son el trabajo propio (ventas informales) con un $15.49 \%$ y otras fuentes con el $11.02 \%$.

\section{- Distribución del Ingreso}

Se les preguntó a los participantes de qué manera distribuyen su dinero, presentándoles una serie de productos para que respondieran si gastan gran parte de su dinero, una pequeña parte de su dinero o si casi nunca gastan su dinero en dichos productos.

En el producto en el cual los niños y adolescentes gastan la mayor parte de su dinero es en ropa (vestuario) con el $39.29 \%$, el siguiente fue golosinas con el $39.06 \%$ y entretenimiento (cine, videojuegos) con el $33.38 \%$.

Los productos en los que gastan una pequeña parte de su dinero son los que tienen que ver con alimentos (ponqués, paquetes) con un $48.78 \%$ y bebidas (jugos, gaseosas) con $52.27 \%$. Los productos en los que casi nunca gastan su dinero los niños y adolescentes es en los que tienen que ver con comunicaciones como lo son el internet y celular (minutos o datos) con los siguientes porcentajes: internet con el 43.48\%, seguido del celular con el 39.12\%. Esto puede deberse a que sus padres asumen el costo de los planes de internet y de telefonía móvil de los cuales los niños también son usuarios finales.

\section{- Preferencia de compra}

REDMARKA UIMA-Universidad de A Coruña - CIECID

Año V, Número 8, (2012), v2 pp. 21-51

http://www.redmarka.org/

ISSN 1852-2300 
A los encuestados se les preguntó con qué personas les gusta ir a comprar determinados productos (ropa, cepillo de dientes, computador, libros y útiles escolares y consolas de videojuegos), y marcar si les gusta ir solos, en compañía de sus amigos o en compañía de sus padres.

A los participantes les gusta ir en compañía de sus padres a comprar productos que tengan que ver con tecnología y con artículos para estudiar, el $71.96 \%$ afirma que le gusta comprar libros y útiles escolares con los papas, y el 68.97\% afirma que le gusta ir a comprar un computador con los padres, seguido de ir con los amigos con un porcentaje de $18.68 \%$ e ir solos con un $12.35 \%$.

A los niños y adolescentes les gusta ir en compañía de sus amigos a comprar productos que tengan relación con su autoimagen (ropa) o con una actividad de socialización con sus amigos (videojuegos). Por ejemplo, el $47.21 \%$ afirma que le gusta comprar ropa en compañía de los amigos, en tanto que el $26.64 \%$ y el $25.15 \%$ afirmaron que les gusta ir solos y en compañía de sus padres respectivamente. Al 44.89\% le gustaría comprar una consola de videojuegos con los amigos, seguido de ir con los papas con un $36.17 \%$ e ir solos con un $19.94 \%$.

Con respecto a la hipótesis relacionada con la posible diferencia significativa en la percepción que tienen los niños y adolescentes con respecto a la influencia que tienen sus amigos frente a sus padres en su comportamiento global, se aplicó una prueba chi-cuadrado entre las variables "Lo que piensan mis amigos es más importante que lo que piensan mis papas", y "Prefiero compartir tiempo con mis amigos en lugar de hacerlo con mis papas". Como se puede apreciar en la Tabla 5 , los niveles de significancia estadística (Chi-cuadrado de Pearson $=0,000$ ) son menores al valor de prueba de 0,05. Por lo tanto, es posible rechazar la hipótesis nula y establecer que efectivamente existe una diferencia entre las variables en mención.

Tabla 5. Prueba de significancia estadística Chi Cuadrado para las variables "Lo que piensan mis amigos es más importante que lo que piensan mis papas", $y$ "Prefiero compartir tiempo con mis amigos en lugar de hacerlo con mis papas".

REDMARKA UIMA-Universidad de A Coruña - CIECID

Año V, Número 8, (2012), v2 pp. 21-51 http://www.redmarka.org/ ISSN 1852-2300 


\begin{tabular}{|c|c|c|c|c|c|}
\hline \multicolumn{6}{|c|}{ Pruebas de chi-cuadrado } \\
\hline & Valor & gl & $\begin{array}{l}\text { Sig. asintótica } \\
\text { (bilateral) }\end{array}$ & $\begin{array}{c}\text { Sig. exacta } \\
\text { (bilateral) }\end{array}$ & $\begin{array}{l}\text { Sig. exacta } \\
\text { (unilateral) }\end{array}$ \\
\hline Chi-cuadrado de Pearson & $56,262^{b}$ & 1 &, 000 & & \\
\hline $\begin{array}{l}\text { Corrección por } \\
\text { continuidad }\end{array}$ & 54,727 & 1 &, 000 & & \\
\hline Razón de verosimilitudes & 54,680 & 1 & ,000 & & \\
\hline $\begin{array}{l}\text { Estadístico exacto de } \\
\text { Fisher }\end{array}$ & & & & ,000 & 000 \\
\hline $\begin{array}{l}\text { Asociación lineal por } \\
\text { lineal }\end{array}$ & 56,208 & 1 & ,000 & & \\
\hline $\mathrm{N}$ de casos válidos & 1033 & & & & \\
\hline
\end{tabular}

a. Calculado sólo para una tabla de $2 \times 2$.

b. 0 casillas $(, 0 \%)$ tienen una frecuencia esperada inferior a 5 . La frecuencia mínima esperada es 42,61 .

Al observar la tabla de contingencia (Ver tabla 6) de las categorías de las variables se puede advertir el hecho de que existe una mayoría (frecuencia observada = $607 ; 58,7 \%$ ) que muestra una percepción que indica claramente una mayor influencia de sus padres en ellos, ya que este porcentaje corresponde a aquellos que mencionaron que lo que piensan sus amigos no es más importante que lo que piensan sus padres y que a su vez prefieren compartir tiempo con sus padres en lugar de hacerlo con sus amigos. Sin embargo, y a pesar de lo anterior, existe un porcentaje importante (frecuencia observada $=314 ; 30,3 \%$ ) que indica por una parte que lo que piensan sus amigos no es más importante que lo que piensan sus padres, al mismo tiempo que afirman que prefieren compartir el tiempo con sus amigos en lugar de hacerlo con sus padres.

Tabla 6. Tabla de contingencia 


\begin{tabular}{|c|c|c|c|c|c|}
\hline & & & \multicolumn{2}{|c|}{$\begin{array}{l}\text { Prefiero compartir } \\
\text { tiempo con mis } \\
\text { amigos en lugar de } \\
\text { hacerlo con mis papas }\end{array}$} & \multirow[b]{2}{*}{ Total } \\
\hline & & & $\mathrm{Si}$ & No & \\
\hline \multirow{4}{*}{$\begin{array}{l}\text { Lo que piensan mis } \\
\text { amigos es más } \\
\text { importante que lo que } \\
\text { piensan mis papas }\end{array}$} & \multirow[t]{2}{*}{$\mathrm{Si}$} & Recuento & 79 & 33 & 112 \\
\hline & & Frecuencia esperada & 42,6 & 69,4 & 112,0 \\
\hline & \multirow[t]{2}{*}{ No } & Recuento & 314 & 607 & 921 \\
\hline & & Frecuencia esperada & 350,4 & 570,6 & 921,0 \\
\hline \multirow[t]{2}{*}{ Total } & & Recuento & 393 & 640 & 1033 \\
\hline & & Frecuencia esperada & 393,0 & 640,0 & 1033,0 \\
\hline
\end{tabular}

Estos resultados indicarían que los niños y adolescentes aunque han sido influenciados en décadas recientes por los medios y la presión social creciente que ejercen los pares, siguen prefiriendo compartir con sus padres en lugar de hacerlo con sus amigos.

En relación con lo anterior se observa un fenómeno interesante al realizar el procedimiento de prueba de la hipótesis relacionada con una posible asociación significativa entre el rango de edad y la percepción que tienen los niños y adolescentes con respecto a la influencia que tienen sus amigos frente a sus padres en su comportamiento global. Como se desprende de los resultados presentados en la Tabla 7, existe una asociación significativa entre las variables rango de edad y "Lo que piensan mis amigos es más importante que lo que piensan mis papas". El valor de significancia (Chi-cuadrado de Pearson $=0,041$ ) es menor al valor de prueba de 0,05.

Tabla 7. Prueba de significancia estadística Chi Cuadrado para las variables "rango de edad" $y$ "Lo que piensan mis amigos es más importante que lo que piensan mis papas" 


\begin{tabular}{|c|c|c|c|c|c|}
\hline \multicolumn{6}{|c|}{ Pruebas de chi-cuadrado } \\
\hline & Valor & $\mathrm{gl}$ & $\begin{array}{l}\text { Sig. asintótica } \\
\text { (bilateral) }\end{array}$ & $\begin{array}{l}\text { Sig. exacta } \\
\text { (bilateral) }\end{array}$ & $\begin{array}{l}\text { Sig. exacta } \\
\text { (unilateral) }\end{array}$ \\
\hline Chi-cuadrado de Pearson & $4,182^{b}$ & 1 & , 041 & & \\
\hline $\begin{array}{l}\text { Corrección por } \\
\text { continuidad }\end{array}$ & 3,722 & 1 &, 054 & & \\
\hline Razón de verosimilitudes & 4,504 & 1 &, 034 & & \\
\hline $\begin{array}{l}\text { Estadístico exacto de } \\
\text { Fisher }\end{array}$ & & & & 049 & ,024 \\
\hline $\begin{array}{l}\text { Asociación lineal por } \\
\text { lineal }\end{array}$ & 4,178 & 1 &, 041 & & \\
\hline $\mathrm{N}$ de casos válidos & 1031 & & & & \\
\hline
\end{tabular}

Al examinar las frecuencias observadas frente a las esperadas en las diferentes celdas que componen la tabla de contingencias de la prueba (Ver Tabla 8), se identifica en primer lugar, que existe una frecuencia observada (19) significativamente menor que la esperada $(27,8)$ para aquellos participantes de entre 9 y 12 años de edad que afirman que lo que dicen sus amigos es más importante que lo que dicen sus papas, en tanto que se evidencia una frecuencia observada (94) significativamente mayor que la esperada $(85,2)$ en los participantes de entre 13 y 17 años que afirman que lo que dicen sus amigos es más importante que lo que dicen sus papas. Es decir, que al aumentar la edad, la influencia de los amigos empieza a cobrar una mayor relevancia en las vidas de los adolescentes.

\section{Tabla 8}

REDMARKA UIMA-Universidad de A Coruña - CIECID

Año V, Número 8, (2012), v2 pp. 21-51

http://www.redmarka.org/

ISSN 1852-2300 
Tabla de contingencia rangos de edad nuevos * Lo que piensan mis amigos es más importante que lo que piensan mis papas

\begin{tabular}{|c|c|c|c|c|c|}
\hline & \multicolumn{2}{|c|}{$\begin{array}{l}\text { Lo que piensan mis } \\
\text { amigos es más } \\
\text { importante que lo que } \\
\text { piensan mis papas }\end{array}$} & \multirow[b]{2}{*}{ Total } \\
\hline & & & $\mathrm{Si}$ & No & \\
\hline \multirow{4}{*}{$\begin{array}{l}\text { rangos de edad } \\
\text { nuevos }\end{array}$} & De 9 a 12 & Recuento & 19 & 235 & 254 \\
\hline & & Frecuencia esperada & 27,8 & 226,2 & 254,0 \\
\hline & De 13 a 17 & Recuento & 94 & 683 & 777 \\
\hline & & Frecuencia esperada & 85,2 & 691,8 & 777,0 \\
\hline \multirow[t]{2}{*}{ Total } & & Recuento & 113 & 918 & 1031 \\
\hline & & Frecuencia esperada & 113,0 & 918,0 & 1031,0 \\
\hline
\end{tabular}

El mismo resultado se observa al aplicar la prueba Chi cuadrado a las variables "rango de edad" y "Prefiero compartir tiempo con mis amigos en lugar de hacerlo con mis papas". Al interpretar los resultados presentados en la Tabla 9, existe una asociación significativa entre las variables, dado que el valor de significancia (Chicuadrado de Pearson $=0,001$ ) es menor al valor de prueba de 0,05.

Tabla 9. Prueba de significancia estadística Chi Cuadrado para las variables "rango de edad" y "Prefiero compartir tiempo con mis amigos en lugar de hacerlo con mis papas"

Pruebas de chi-cuadrado

\begin{tabular}{|c|c|c|c|c|c|}
\hline & Valor & gl & $\begin{array}{l}\text { Sig. asintótica } \\
\text { (bilateral) }\end{array}$ & $\begin{array}{l}\text { Sig. exacta } \\
\text { (bilateral) }\end{array}$ & $\begin{array}{l}\text { Sig. exacta } \\
\text { (unilateral) }\end{array}$ \\
\hline Chi-cuadrado de Pearson & $11,937^{b}$ & 1 & ,001 & & \\
\hline $\begin{array}{l}\text { Corrección par } \\
\text { continuidad }\end{array}$ & 11,429 & 1 & 001 & & \\
\hline Razón de verosimilitudes & 12,257 & 1 & ,000 & & \\
\hline $\begin{array}{l}\text { Estadístico exacto de } \\
\text { Fisher }\end{array}$ & & & & ,001 & ,000 \\
\hline $\begin{array}{l}\text { Asociación lineal por } \\
\text { lineal }\end{array}$ & 11,925 & 1 & ,001 & & \\
\hline $\mathrm{N}$ de casos válidos & 1028 & & & & \\
\hline
\end{tabular}

a. Calculado sólo para una tabla de $2 \times 2$.

b. 0 casillas $(, 0 \%)$ tienen una frecuencia esperada inferior a 5 . La frecuencia mínima esperada es 97,24 .

Si se contrastan las frecuencias observadas frente a las esperadas en las diferentes celdas que componen la tabla de contingencias de la prueba (Ver Tabla 10), se identifica en primer lugar, que existe una frecuencia observada (74)

REDMARKA UIMA-Universidad de A Coruña - CIECID

Año V, Número 8, (2012), v2 pp. 21-51

http://www.redmarka.org/

ISSN 1852-2300 
significativamente menor que la esperada $(97,2)$ para aquellos participantes de entre 9 y 12 años de edad que afirman que prefieren compartir tiempo con sus amigos en lugar de hacerlo con sus papas, en tanto que se observa una frecuencia observada (318) significativamente mayor que la esperada $(294,8)$ en los participantes de entre 13 y 17 años que afirman que prefieren compartir tiempo con sus amigos en lugar de hacerlo con sus papas. Es decir, que al aumentar la edad los adolescentes indican tener una creciente preferencia por compartir más tiempo con sus amigos, frente a la opción de compartir con sus padres, la cual sigue siendo marcadamente fuerte en el rango de edad que va de los 9 a los 12 años.

\section{Tabla 10}

Tabla de contingencia rangos de edad nuevos * Prefiero compartir tiempo con mis amigos en lugar de hacerlo con mis papas

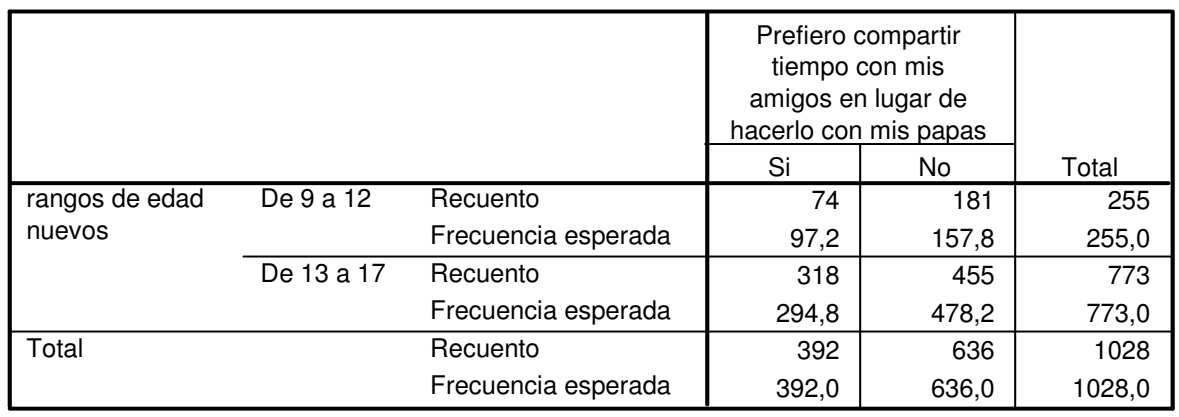

Estos resultados en conjunto indican que los niños y adolescentes tienen no solo una capacidad de compra creciente sino cada vez también una mayor independencia como personas y como consumidores en comparación con generaciones anteriores, lo cual se puede evidenciar en el efecto progresivo de la importancia que han venido adquiriendo los pares (amigos) en las preferencias de los adolescentes en particular.

\section{Discusión}

La investigación del consumidor infantil ha ganado en las últimas décadas no solo un incremento tanto en el volumen de estudios realizados como en la variedad de 
posturas teóricas que se han formulado desde diferentes disciplinas para interpretar este importante fenómeno social y económico. Lo primero que se debe resaltar a partir de los resultados obtenidos en el presente estudio, es la creciente relación que se evidencia entre los niños y adolescentes y las marcas presentes en el mercado en una cantidad cada vez mayor de categorías de producto. En concordancia con lo que menciona John (1999), los niños están incorporando a una edad cada vez más temprana no solo una mayor independencia con respecto a las decisiones de compra en diferentes contextos, sino que en estos procesos de conformación de sus patrones de compra y preferencias de marca juegan un papel más decisivo algunos factores externos tales como sus pares y las acciones de mercadeo a las que están expuestos de forma masiva a través de los medios de comunicación tanto tradicionales como alternativos. Según los resultados, en el rango de edad que va de los 13 a los 17 años, los adolescentes prefieren la compañía de sus amigos, en lugar de la de sus padres, lo cual apoya el supuesto de que en estas edades se promueve un mejor entendimiento de las relaciones interpersonales, y las marcas parecen estar en medio de estos procesos de presión social entre los miembros de los grupos de referencia debido a la fuerte presencia en los medios de comunicación más consumidos por ellos, particularmente el internet. Sin embargo, para el rango de edad comprendido entre los 9 y 12 años, la familia parece tener un rol preponderante, ya que como lo menciona Sidin et al (2008), en este ámbito los mecanismos de modelamiento de los padres son más influyentes debido a que aún los niños en esta edad tienen una mayor cantidad de interacción directa con ellos, tal como se mencionó en los resultados concernientes a las pruebas relacionadas con la edad y la intención de compartir más tiempo con los amigos o con los padres. Los niños en este rango de edad muestran una clara tendencia a preferir la compañía de sus padres en lugar de la de sus amigos. Además, como se pudo observar en el Análisis de Correspondencias Simples, las marcas, en particular aquellas relacionadas con productos tecnológicos y páginas de internet están muy presentes en el perfil léxico de los dos grupos de edad analizados. Las marcas que se encuentren 
presentes en estos nuevos contextos digitales y que se relacionen directamente con procesos de socialización con sus pares tendrán una mayor oportunidad de crecimiento en este grupo poblacional. Esto se desprende de los resultados concernientes a los perfiles léxicos tanto de cada grupo de edad como del que comparten todos los participantes de la muestra. En ellos se destacan formas léxicas como "internet”, "celular", "chatear”, "descargar”, “con amigos”, "Google”, "Facebook", lo cual brinda una clara alusión a que su realidad y su cotidianidad en el consumo y la socialización esta mediada por estas nuevas formas de interacción social digital. Aunque estos procesos de apropiación de las marcas presentes en internet es compartida por los dos grupos de edad, en aquellos de entre 9 y 12 años, siguen estando presentes de manera significativa las actividades que comparten todavía con sus padres tales como el ir de compras al supermercado y ver noticias y novelas en televisión. Además, los resultados dejan ver que en un país en vías de desarrollo como Colombia, estos procesos de adopción de las nuevas tecnologías llevan un paso acelerado y probablemente se están convirtiendo, de acuerdo con la clasificación dada por Churchill and Moschis (1979), en el factor ambiental que está modificando en mayor medida el tipo, cantidad y calidad de la información y las experiencias de compra que están teniendo los niños y adolescentes en los contextos de consumo de la actualidad. El consumo de estos nuevos medios puede estar incrementando el rol influenciador de los amigos, sobre todo si sobre estos grupos a su vez, recae la masiva presencia de las marcas que sugieren modelos de consumo cada vez más exigentes y complejos, al tiempo que los adolescentes tienen una mayor independencia para poder escoger marcas que les permitan reafirmar su propia individualidad, así esta sea moldeada por las mismas marcas que los rodean en sus espacios cotidianos. Estos resultados concuerdan en gran medida con las grandes tendencias tanto empíricas como teóricas que han venido aportando diferentes autores en las últimas décadas y que indican que la velocidad con la cual se están dando los cambios en los procesos de socialización del consumo en los niños y adolescentes requiere de una constante revisión y actualización tanto 
de las metodologías de estudio como de las posibilidades de análisis de datos. En este sentido, se propone con este estudio una alternativa para el abordaje de esta población con el Análisis de Tipologías Lingüísticas, ya que puede ser muy enriquecedor el analizar la producción verbal de los niños desde un enfoque de carácter más cualitativo. Estos esfuerzos por brindar otros puntos de vista son necesarios en la medida en que el entendimiento y la comprensión del fenómeno del consumo infantil requiere una mirada interdisciplinaria dada su alta complejidad en términos de lo psicológico, lo social y lo económico.

\section{Referencias Bibliográficas}

Center for a New American Dream. (2002). Just Facts About Advertising and Marketing to Children. Consultado el 01/06/2011 en: www.newdream.org/campaign/kids/facts.html

Chandler, T., Heinzerling, B. (1999). Children and adolescents in the market place: Twenty-five years of academic research. Ann Arbor: The Pierian Press.

Churchill, G., Moschis, G. (1979). Television and Interpersonal Influences on Adolescent Consumer Learning. Journal of Consumer Research, 6 , pp. 2335.

Dotson, M., Hyatt, E., (1994). The impact of changes in the household on the consumer socialization process. Proceedings of the Southern Marketing Association. New Orleans, November, pp. 156-160.

Dotson, M., Hyatt, E., (2000). A comparison of the parents and children knowledge of brands and advertising slogans in the United States: implications for consumer socialization. Journal of Marketing Communications. 2, 4, pp. 219 -230 .

Dotson, M., Hyatt, E., (2005). Major influence factors in children's consumer socialization. The Journal of Consumer Marketing, 22, 1, pp 35-42. 
Grassl, W. (1999). The reality of brands: towards an ontology of marketing. American Journal of Economis and Sociology. 58, 2, pp. 313 - 360.

John, D. (1999). Consumer Socialization of Children: A Retrospective Look at Twenty-Five Years of Research. Journal of Consumer Research, 26, 1, pp. 183-213.

(2008). Stages of Consumer Socialization: The Development of Consumer Knowledge, Skills, and Values from Childhood to Adolescence," in Handbook of Consumer Psychology, ed. Curtis P. Haugtvedt, Paul M. Herr, and Frank R. Kardes, New York: Psychology, 221- 246.

Keller, K.L. (1998). Strategic Brand Management. Prentice Hall, Upper Saddle River, NJ.

McNeal, J.U., Yeh, C.H. (1993). Born to Shop. American Demographics, June, pp. $34-39$.

Moschis, G., Churchill, G. (1978). Consumer soclialization: a theoretical and empirical analysis. Journal of Merketing Research. 15, November, pp. 599609.

Moschis, G., Moore, R. (1979). Decision Making among the Young: A Socialization Perspective. Journal of Consumer Research, 6 (September), pp. 101-112.

Moschis, G., Moore, R. (1980). Purchasing Behavior of adolescent consumers. Proceedings of the American Marketing Association. 45, pp. 89 - 92.

Moschis, G. (1981), Patterns of consumer learning, Journal of the Academy of Marketing Science, Vol. 9, pp. 110-124.

Ozgen, O. (2003). An analysis of child consumers in Turkey. International Journal of Consumer Studies, 27, 5, pp. 366-80.

Pecora, N. (1995). Children and television advertising from a social science perspective. Critial Studies in Mass Communication, 12, 3, pp. 354 - 365.

Public Broadcasting Service (1999). The Merchants of Cool. Frontline, PBS Video, Public Boradcasting Service, Alexandria.

Robayo, O. (2011). La Investigación del Consumidor Infantil: Hallazgos y Perspectivas de Estudio. En prensa. 
Schulman, Y., Clancy, K. (1992). Adults and children, a real gap. Adweek. 33, February 10, p. 48.

Shimp, T. (2000). Advertising and promotion: supplemental aspects of integrated marketing communications. The Dryden Press. Forth Worth.

Sidin, S., Rashid, Z., (2008). Effects of social variables on urban children's consumption attitude and behavior intentions. The Journal of Consumer Marketing, 25, 1, pp 7-15.

Solomon, D. (1991). The Latest in Children's Research: How to Use It More Effectively. in Transcript Proceedings: Third ARF Children s Research Workshop, New York: Advertising Research Foundation.

Ward, S. (1974). Consumer socialization. Journal of Consumer Research, 1, September, pp. 1 - 14.

Weehsler, P. (1997). Hey kid, buy this!. Business Week. June 30, pp. $62-67$.

Wimalasiri, J., (2004). A cross-national study on children's purchasing behavior and parental response. The Journal of Consumer Marketing. 21, 4. pp. 274 $-284$.

Wolff, C. (2003). Decrying how marketers get their hooks into teens. Boston Globe Online. February 18. Consultado en: www.boston.com/dailyglobe2/047/

\section{Para citar este artículo}

Pinzón, Oscar Javier (20-06-2012). LAS MARCAS Y EL PROCESO DE SOCIALIZACIÓN DEL CONSUMO EN NIÑOS Y ADOLESCENTES: EL CASO COLOMBIANO.

REDMARKA - CIECID - Unidad de Investigación en Marketing Aplicado-Universidad de A Coruña Año V, Número 8, V2, pp.21-51

ISSN $1852-2300$

URL del Documento : cienciared.com.ar/ra/doc.php? $n=1664$

URL de la Revista : cienciared.com.ar/ra/revista. $p h p ?$ wid=39

REDMARKA UIMA-Universidad de A Coruña - CIECID 